Louisiana State University

LSU Digital Commons

3-10-2011

\title{
Astrophysical parameters and habitable zone of the exoplanet hosting star GJ 581
}

\author{
Kaspar Von Braun \\ California Institute of Technology \\ Tabetha S. Boyajian \\ Georgia State University \\ Stephen R. Kane \\ California Institute of Technology \\ Gerard T. Van Belle \\ European Southern Observatory \\ David R. Ciardi \\ California Institute of Technology
}

See next page for additional authors

Follow this and additional works at: https://digitalcommons.Isu.edu/physics_astronomy_pubs

\section{Recommended Citation}

Von Braun, K., Boyajian, T., Kane, S., Van Belle, G., Ciardi, D., López-Morales, M., McAlister, H., Henry, T., Jao, W., Riedel, A., Subasavage, J., Schaefer, G., Ten Brummelaar, T., Ridgway, S., Sturmann, L., Sturmann, J., Mazingue, J., Turner, N., Farrington, C., Goldfinger, P., \& Boden, A. (2011). Astrophysical parameters and habitable zone of the exoplanet hosting star GJ 581. Astrophysical Journal Letters, 729 (2 PART II) https://doi.org/10.1088/2041-8205/729/2/L26

This Article is brought to you for free and open access by the Department of Physics \& Astronomy at LSU Digital Commons. It has been accepted for inclusion in Faculty Publications by an authorized administrator of LSU Digital Commons. For more information, please contact ir@lsu.edu. 


\section{Authors}

Kaspar Von Braun, Tabetha S. Boyajian, Stephen R. Kane, Gerard T. Van Belle, David R. Ciardi, Mercedes López-Morales, Harold A. McAlister, Todd J. Henry, Wei Chun Jao, Adric R. Riedel, John P. Subasavage, Gail Schaefer, Theo A. Ten Brummelaar, Stephen Ridgway, Laszlo Sturmann, Judit Sturmann, Jude Mazingue, Nils H. Turner, Chris Farrington, P. J. Goldfinger, and Andrew F. Boden 


\title{
ASTROPHYSICAL PARAMETERS AND HABITABLE ZONE OF THE EXOPLANET HOSTING STAR GJ 581
}

\author{
Kaspar von Braun ${ }^{1}$, Tabetha S. Boyajian ${ }^{2,10}$, Stephen R. Kane ${ }^{1}$, Gerard T. van Belle ${ }^{3}$, David R. Ciardi ${ }^{1}$, \\ Mercedes López-Morales ${ }^{4,5}$, Harold A. McAlister ${ }^{2}$, Todd J. Henry ${ }^{2}$, Wei-Chun JaO ${ }^{2}$, Adric R. Riedel ${ }^{2}$, \\ John P. Subasavage ${ }^{6}$, Gail Schaefer ${ }^{7}$, Theo A. Ten BrummelaAr ${ }^{7}$, Stephen Ridgway $^{8}$, Laszlo SturmanN $^{7}$, \\ Judit Sturmann ${ }^{7}$, Jude Mazingue ${ }^{7}$, Nils H. Turner ${ }^{7}$, Chris Farrington ${ }^{7}$, P. J. Goldfinger ${ }^{7}$, And Andrew F. Boden ${ }^{9}$ \\ ${ }^{1}$ NASA Exoplanet Science Institute, California Institute of Technology, MC 100-22, Pasadena, CA 91125, USA; kaspar@ caltech.edu \\ ${ }^{2}$ Center for High Angular Resolution Astronomy and Department of Physics and Astronomy, Georgia State University, P.O. Box 4106, Atlanta, \\ GA 30302-4106, USA \\ ${ }^{3}$ European Southern Observatory, Karl-Schwarzschild-Str. 2, 85748 Garching, Germany \\ ${ }^{4}$ Institut de Ciències de L'Espai (CSIC-IEEC), Campus UAB, Facultat Ciències, Torre C5 parell 2, 08193 Bellaterra, Barcelona, Spain \\ 5 Department of Terrestrial Magnetism, Carnegie Institution of Washington, 5241 Broad Branch Road, NW, Washington, DC 20015, USA \\ ${ }^{6}$ Cerro Tololo Inter-American Observatory, Casilla 603, La Serena, Chile \\ ${ }^{7}$ The CHARA Array, Mount Wilson Observatory, Mount Wilson, CA 91023, USA \\ ${ }^{8}$ National Optical Astronomy Observatory, P.O. Box 26732, Tucson, AZ 85726-6732, USA \\ ${ }^{9}$ California Institute of Technology, Pasadena, CA 91125, USA \\ Received 2010 December 21; accepted 2011 February 1; published 2011 February 16
}

\begin{abstract}
GJ 581 is an M dwarf host of a multiplanet system. We use long-baseline interferometric measurements from the CHARA Array, coupled with trigonometric parallax information, to directly determine its physical radius to be $0.299 \pm 0.010 R_{\odot}$. Literature photometry data are used to perform spectral energy distribution fitting in order to determine GJ 581's effective surface temperature $T_{\mathrm{EFF}}=3498 \pm 56 \mathrm{~K}$ and its luminosity $L=0.01205 \pm 0.00024 L_{\odot}$. From these measurements, we recompute the location and extent of the system's habitable zone and conclude that two of the planets orbiting GJ 581, planets $\mathrm{d}$ and $\mathrm{g}$, spend all or part of their orbit within or just on the edge of the habitable zone.
\end{abstract}

Key words: infrared: stars - planetary systems - stars: fundamental parameters - stars: individual (GJ 581) - stars: late-type - techniques: interferometric

Online-only material: color figure

\section{INTRODUCTION}

The formation, evolution, and environment of extrasolar planets are heavily dependent on the astrophysical properties of their respective parent stars. In particular, the location and extent of the system's habitable zone (HZ) are direct functions of the parent star's size and surface temperature, both of which are frequently determined by stellar modeling. The advent of long-baseline interferometry at wavelengths in the near-infrared or optical range has made it possible to directly measure these stellar astrophysical properties (e.g., Baines et al. 2007, 2008, 2009, 2010; van Belle \& von Braun 2009, and references therein).

$\mathrm{M}$ dwarfs are popular targets for exoplanet searches, in part due to their low intrinsic luminosity, which makes it possible for planets with orbital periods of much less than a year to be located near or inside the system's HZ. GJ 581 (= HIP 74995, $V=10.570, H=6.095, K=5.837$ ) is a nearby M2-3 dwarf (Bessel 1990; Henry et al. 1994; Hawley et al. 1996; Cutri et al. 2003) that hosts six currently reported planets, all detected by the radial velocity (RV) technique and successively announced in Bonfils et al. (2005, Neptune-mass planet b), Udry et al. (2007, super-Earth mass planets c and d), Mayor et al. (2009, Earthmass planet e), and most recently, Vogt et al. (2010, super-Earth mass planets $f$ and $g$ ).

Here we present the fundamental astrophysical parameters of GJ 581 primarily based on interferometric observations, and comment on the resulting location and extent of the system's HZ

\footnotetext{
${ }^{10}$ Hubble Fellow.
}

as well as the locations of the orbiting planets with respect to it. Our observations are described in Section 2, and the calculations of the resulting stellar astrophysical parameters in Section 3. We discuss the HZ of the GJ 581 system in Section 4, and summarize and conclude in Section 5.

\section{OBSERVATIONS}

\subsection{Interferometry}

Our interferometric observations of GJ 581 were carried out using the Georgia State University Center for High Angular Resolution Astronomy (CHARA) Array (ten Brummelaar et al. 2005) in the context of ongoing surveys to determine fundamental parameters of exoplanet hosting stars (von Braun et al. 2010) and of low-mass stars (Boyajian et al. 2010). We used the single baseline CHARA Classic beam combiner in the $H$ band $\left(\lambda_{\text {central }}=1.67 \mu \mathrm{m}\right)$ and collected data on six nights between 2010 March and June. We observed with CHARA's longest baselines S1/E1 ( $330 \mathrm{~m}, 19$ observations) and W1/ E1 ( $313 \mathrm{~m}$, eight observations). The calibrator stars HD $136257\left(V=7.55, H=6.24, \theta_{\mathrm{SED}}=0.233 \pm 0.007 \mathrm{mas}^{11}\right)$ and $\mathrm{HD} 136713\left(V=7.99, H=5.83, \theta_{\mathrm{SED}}=0.323 \pm 0.023\right.$ mas) were observed along with GJ 581 in bracketed sequences. These calibrator stars were chosen to be near-point-like sources of similar $\mathrm{H}$-band magnitudes as GJ 581 and located at small angular distances from it.

The uniform disk and limb-darkened angular diameters $\left(\theta_{\mathrm{UD}}\right.$ and $\theta_{\mathrm{LD}}$, respectively; see Table 1) are found by fitting the

\footnotetext{
${ }^{11} \theta_{\mathrm{SED}}$ corresponds to the estimated angular diameter based on spectral energy distribution fitting.
} 


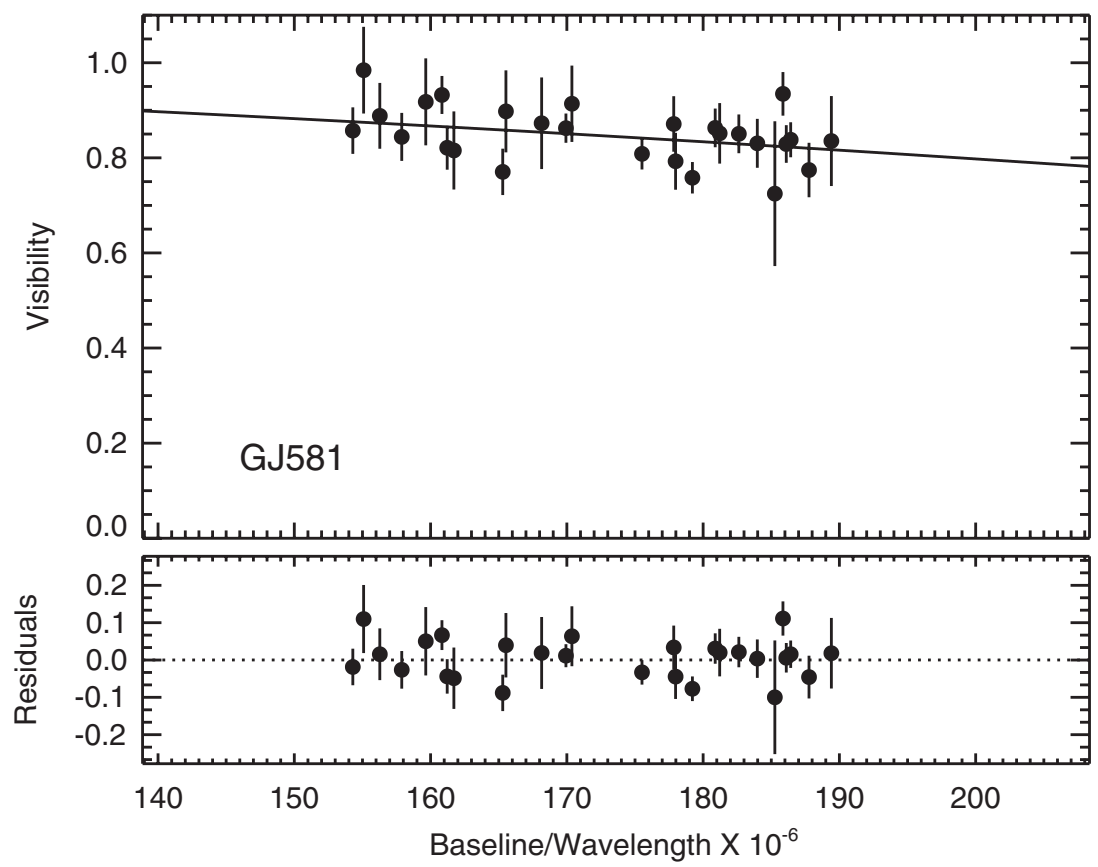

Figure 1. Calibrated visibility observations along with the limb-darkened angular diameter fit for GJ 581. For details, see Section 2.1.

Table 1

Stellar Properties of GJ 581

\begin{tabular}{lcc}
\hline \hline Parameter & Value & Reference \\
\hline Spectral type & M $2.5 \mathrm{~V}$ & Henry et al. (1994) \\
$V-K$ & $4.733 \pm 0.083$ & Bessel (1990); Cutri et al. (2003) \\
{$[\mathrm{Fe} / \mathrm{H}]$} & -0.135 & Average $^{\mathrm{a}}$ \\
$\theta_{\mathrm{UD}}(\mathrm{mas})$ & $0.433 \pm 0.014$ & This work \\
$\theta_{\mathrm{LD}}(\operatorname{mas})$ & $0.446 \pm 0.014$ & This work \\
Parallax (mas) & $160.12 \pm 1.33$ & This work \\
Radius $\left(R_{\odot}\right)$ & $0.299 \pm 0.010$ & This work \\
Luminosity $\left(L_{\odot}\right)$ & $0.01205 \pm 0.00024$ & This work \\
$T_{\mathrm{EFF}}(\mathrm{K})$ & $3498 \pm 56$ & This work \\
$\log g^{\mathrm{b}}$ & $4.96 \pm 0.08$ & This work \\
\hline
\end{tabular}

Notes. Properties of GJ 581. For details, see Section 3.

a Average from Bonfils et al. (2005), Johnson \& Apps (2009), Rojas-Ayala et al. (2010), and Schlaufman \& Laughlin (2010).

b The surface gravity $\log g$ is based on our quoted values of mass and radius, and its uncertainty is calculated from our empirically determined radius uncertainty. We assume $\sigma M=0.05 M_{\odot} \simeq 16 \%$, which represents a slightly more conservative approach than the $(\sigma M)_{\max }=10 \%$ requirement in the calculation of the $M-L$ relation in Delfosse et al. (2000).

calibrated visibility measurements (Figure 1) to the respective functions for each relation. These formulae may be described as $n$ th-order Bessel functions that are dependent on the angular diameter of the star, the projected distance between the two telescopes, and the wavelength of observation (see Equations (2) and (4) of Hanbury Brown et al. 1974). We use the $H$-band limb-darkening coefficient $\left(\mu_{H}=0.358\right)$ calculated from the PHOENIX model atmospheres code for the corresponding value of $T_{\mathrm{EFF}}=3500 \mathrm{~K}$ and $\log g=5.0$, as tabulated in Claret (2000). ${ }^{12}$ The solutions and their respective errors are computed using MPFIT, a nonlinear least-squares fitting routine in IDL

12 We note that an uncertainty in an assumed parameter will only modestly influence the value of the coefficient, and consequentially the value of $\theta_{\mathrm{LD}}$. For example, changing the model $T_{\mathrm{EFF}}$ by $\pm 300 \mathrm{~K}$ will change the resulting diameter by only a few tenths of a percent, well within our error budget.
(Markwardt 2009). For this solution we find $\chi_{\text {reduced }}^{2}=0.65$, implying that the standard CHARA reduction pipeline may have overestimated our measurement errors. Thus, the results presented here assume a true $\chi_{\text {reduced }}^{2}=1$ to remedy effects of the overestimated uncertainties. Our results are shown in Table 1.

Finally, our interferometric data allowed for a search for separated fringe packets to comment on the potential existence of a previously unresolved stellar companion in a near face-on orbit (see discussion in Baines et al. 2010 and Farrington et al. 2010), which would influence the interpretation of the RV curves. The fringe packets from two stellar sources would overlap for small projected separations of $\sim 0.5-5$ mas, producing a periodic modulation in the angular diameter visibility curve (e.g., Baines et al. 2010). The amplitude of this modulation is set by the flux ratio of the binary components. Based on the range of visibility residuals in our angular diameter fit, we can rule out companions with magnitude differences $\delta H<2.4 \mathrm{mag}$. The fact that we did not find any evidence of a secondary fringe packet (and thus stellar-mass companion at low inclination angles) reinforces the dynamical stability argument of coplanar orbits in this system as described in Mayor et al. (2009) and Beust et al. (2008) by providing an empirical lower-limit constraint on the orbital inclinations of the planetary orbits.

\subsection{Astrometry}

Translating the measured angular radius of GJ 581 into a linear radius depends on its distance. Trigonometric parallax measurements are available for GJ 581 in The General Catalogue of Trigonometric Stellar Parallaxes (van Altena et al. 1995, $\pi_{\text {trig }}=157.9 \pm 5.6$ mas), from van Leeuwen's reduction of the HIPPARCOS space mission observations (van Leeuwen 2008, $\pi_{\text {trig }}=160.91 \pm 2.62$ mas), and from the RECONS group ${ }^{13}$ of

\footnotetext{
13 www.recons.org
} 


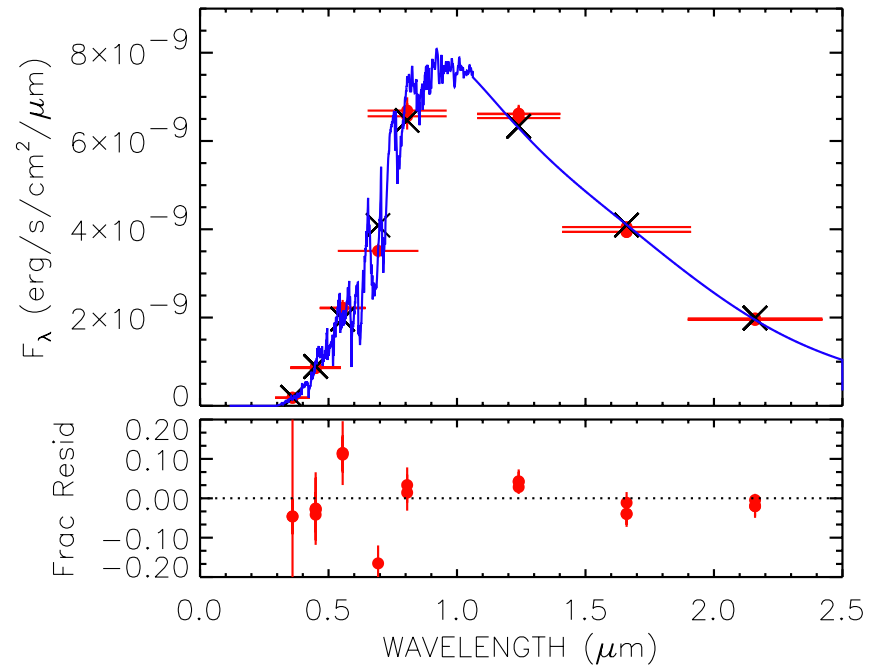

Figure 2. Spectral energy distribution fit for GJ 581. Literature photometry data are plotted on top of the spectral template. Horizontal error bars correspond to filter bandwidths. Crosses indicate flux levels of the spectral template integrated over the filter bandwidth. The fractional residuals are given in the bottom panel, along with scaled error bars in the respective photometry data point. The large $U$-band error bar is due to uncertainties associated with literature photometry. For details, see Section 3 and Table 1.

(A color version of this figure is available in the online journal.)

$\pi_{\text {trig }}=160.01 \pm 1.61$ mas. ${ }^{14}$ The weighted-mean parallax of the three independent measurements for the GJ 581 system is $160.12 \pm 1.33$ mas, driven primarily by the improved RECONS parallax, and is used here in the calculation of GJ 581's physical size.

\section{FUNDAMENTAL ASTROPHYSICAL PARAMETERS OF THE HOST STAR GJ 581}

A summary of our results of the fundamental parameters of GJ 581 is given in Table 1. We describe below how individual values were obtained.

Based on GJ 581's $\pi_{\text {trig }}$ and interferometrically measured angular diameter $\theta_{\mathrm{LD}}$, we calculate its linear radius to be $R=0.299 \pm 0.010 R_{\odot}$. Following the procedure outlined in Section 3.1 of van Belle et al. (2007), we produce a fit of the stellar spectral energy distribution (SED) to the literature UBVRIJHK photometry. We obtain a value of GJ 581's stellar bolometric flux $F_{\mathrm{BOL}}=(9.92 \pm 0.104) \times 10^{-9} \mathrm{erg} \mathrm{cm}^{-2} \mathrm{~s}^{-1}$ and luminosity $L=0.01205 \pm 0.00024 L_{\odot}$. Photometry for our SED fit from the following papers was used along with spectral templates from Pickles (1998): Weis (1993), Bessel (1990), Koen et al. (2002), Cutri et al. (2003, 2MASS $\left.J H K_{s}\right)$, Nordström et al. (2004), Kilkenny et al. (2007), Rayner et al. (2009), and Koen et al. (2010). The transformation from the SAAO photometry system to 2MASS was based on the equations in Koen et al. (2007). We show our SED fit in Figure 2.

It should be noted that, in order to minimize the $\chi^{2}$ of our SED fit, we obtain a non-zero value for extinction $\left(A_{V}=\right.$ $0.174 \pm 0.021)$, which is unexpected for a star at a distance of around $6 \mathrm{pc}$. Forcing $A_{V}=0$ degrades the $\chi^{2}$ from around 2 to around 6 . The associated difference in the calculated value

\footnotetext{
14 This supersedes the value reported in Jao et al. (2005) where details about the RECONS astrometry program at the CTIO $0.9 \mathrm{~m}$ can be found. In essence, the improved parallax results from a reduction of 224 total frames of the GJ 581 field taken over 9.93 years, whereas the previous result was based on 122 frames over 2.95 years.
}

of $F_{\mathrm{BOL}}$ is around $6.7 \%$, corresponding to a $1.6 \%$ effect in the calculation of $T_{\mathrm{EFF}}$ (see Equation (1)) in the sense that an $A_{V}>0$ produces a slightly higher $T_{\mathrm{EFF}}$. We postulate that the extinction could be circumstellar in origin. ${ }^{15}$ Equivalent SED fitting using NextGen synthetic templates (Hauschildt et al. 1999) instead of the empirical Pickles (1998) templates yielded near-identical values of $F_{\mathrm{BOL}}$ and $A_{V}$ for the best-fit templates using $T_{\mathrm{EFF}}=3300 \mathrm{~K}, \log g=5.0$, and $[\mathrm{Fe} / \mathrm{H}]=-0.5$.

The effective temperature $T_{\mathrm{EFF}}$ is calculated based on the rearranged form of the Stefan-Boltzmann equation

$$
T_{\mathrm{EFF}}(\mathrm{K})=2341\left(F_{\mathrm{BOL}} / \theta_{\mathrm{LD}}^{2}\right)^{\frac{1}{4}}
$$

where $F_{\mathrm{BOL}}$ is in units of $10^{-8} \mathrm{erg} \mathrm{cm}^{-2} \mathrm{~s}^{-1}$ and the angular diameter $\theta_{\mathrm{LD}}$ is in units of mas. We calculate GJ 581's effective temperature to be $T_{\mathrm{EFF}}=3498 \pm 56 \mathrm{~K}$.

We derive a mass estimate of $M \simeq 0.3 M_{\odot}$ from the $K$-band mass-luminosity relation in Delfosse et al. (2000). Finally, GJ 581 's mass and radius produce $\log g=4.96 \pm 0.08$, assuming a mass uncertainty of $0.05 M_{\odot}$. See Table 1 for a summary of our results.

The most recent values for GJ 581's astrophysical properties before the ones presented here are the ones calculated in Bonfils et al. (2005). They were subsequently adopted by the papers documenting the discoveries of the other five planets orbiting GJ 581 (Udry et al. 2007; Mayor et al. 2009; Vogt et al. 2010). Below we compare these values to ours.

Bonfils et al. (2005) use a $V$-band bolometric correction from Delfosse et al. (1998) to derive $L=0.013 L_{\odot}$, which constitutes a $4 \sigma$ increase with respect to our value, using only our error estimates; theirs are not given. This discrepancy decreases only marginally (to $3.6 \sigma$ ) if we set equal the two slightly different

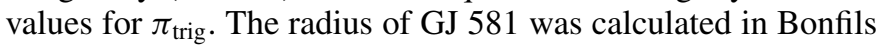
et al. (2005) by applying their estimated mass, $M=0.31 M_{\odot}$, to the mass-radius relation in Chabrier \& Baraffe (2000). We find that their resulting value of $0.29 R_{\odot}$ is $\sim 1 \sigma$ (or $\sim 3.0 \%$ ) smaller than our direct measurement. Finally, the estimate of $T_{\mathrm{EFF}}$ in Bonfils et al. (2005), based on their calculated stellar radius and luminosity, is $3622 \mathrm{~K}$, which is $3.5 \%(2.2 \sigma)$ higher than our value.

Interestingly, some previous estimates of GJ 581's $T_{\mathrm{EFF}}$ yield results with lower temperature estimates. The temperature calibration for M dwarfs presented in Bessell (1995) implies a value for GJ 581's $T_{\mathrm{EFF}}$ of $3320 \mathrm{~K}$. Furthermore, two different approaches used in Casagrande et al. (2008) result in $T_{\mathrm{EFF}}=$ $3320 \mathrm{~K}$ and $T_{\mathrm{EFF}}=3300 \mathrm{~K}$, respectively. Conversely, the (independently calculated) empirical relation between $T_{\mathrm{EFF}}$ and $(V-K)_{0}$ in van Belle \& von Braun (2009, Equation (2)) produces an effective temperature of GJ 581 of just over $3500 \mathrm{~K}$.

\section{THE HABITABLE ZONE IN THE GJ 581 SYSTEM}

A system's traditional $\mathrm{HZ}$ is defined as the range of circumstellar distances from a star within which a planet could have liquid water on its surface, given a dense enough atmosphere. The various criteria for defining the $\mathrm{HZ}$ are described in detail in Kasting et al. (1993) and further generalized by Underwood et al. (2003). For the GJ 581 system, the HZ boundaries were

\footnotetext{
15 Both Kóspál et al. (2009) and Bryden et al. (2009) report a $70 \mu \mathrm{m}$ excess in the analysis of Spitzer MIPS archival data of around three times the value expected from extrapolating the stellar SED to wavelengths redward of 2MASS photometry, although Bryden et al. (2009) classify the significance of their detection as marginal.
} 

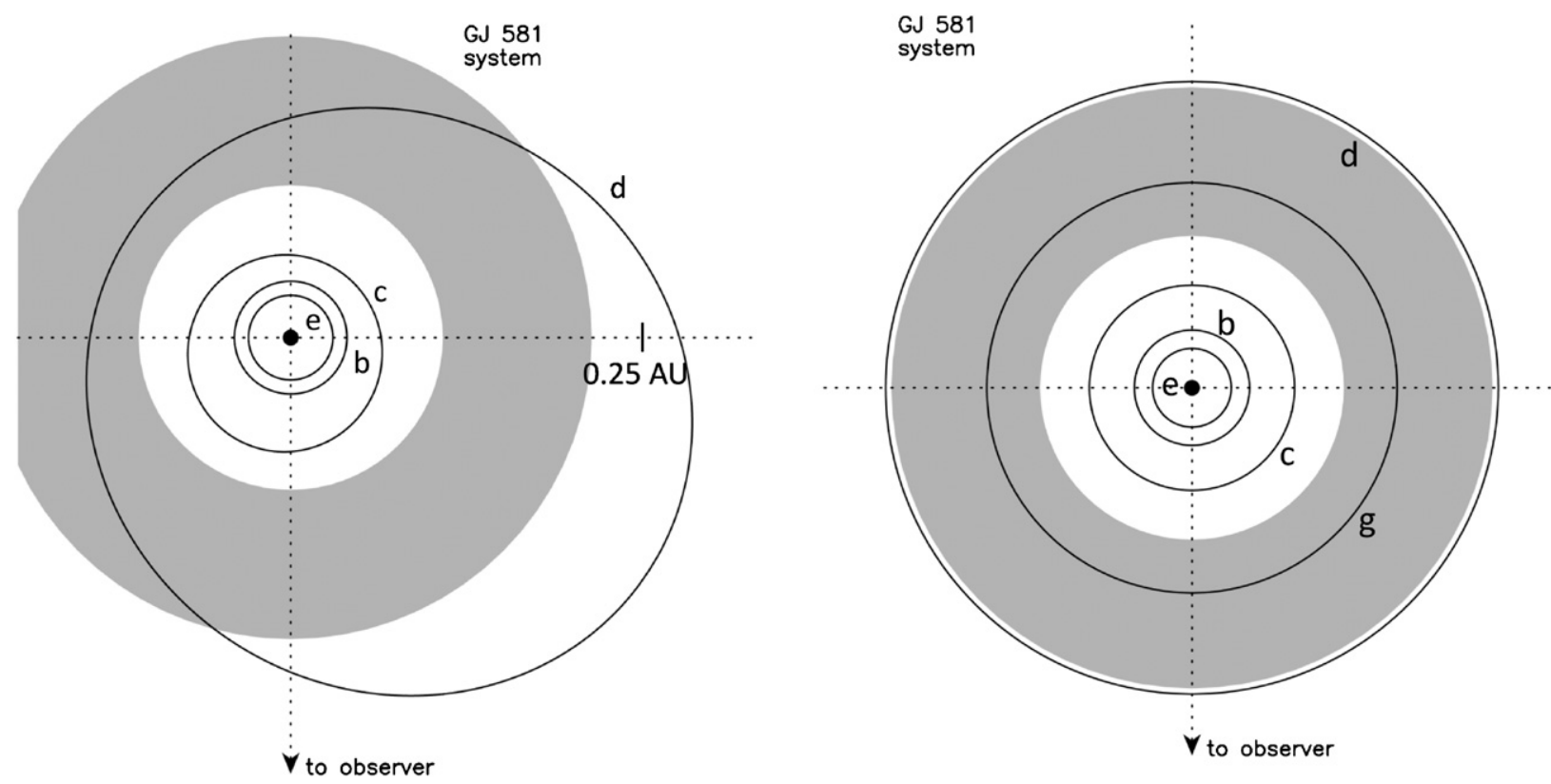

Figure 3. Top-down view of the GJ 581 system. The HZ is indicated by the gray shaded region with calculated boundaries of 0.11 and 0.21 AU. Left panel: scenario 1 adopted from Mayor et al. (2009). Planet d spends part of its elliptical orbit in the HZ. Right panel: scenario 2 adopted from Vogt et al. (2010). Planet $g$ spends all its orbits inside the HZ, which planet d orbits right on its outer edge. Planet $\mathrm{f}(a=0.758 \mathrm{AU})$ is not shown for purpose of clarity. For details, see Section 4 and Table 2.

calculated by Udry et al. (2007), Mayor et al. (2009), and Vogt et al. (2010) in the context of the respective detections of additional planets in the GJ 581 system. A detailed analysis of the habitability of the system under various assumptions can be found in Selsis et al. (2007).

Our recalculation of the boundaries of the GJ 581's HZ based on our directly determined host star properties is based on the equations in Underwood et al. (2003) and Jones \& Sleep (2010) that relate the radii of the inner and outer edges of the HZ to the luminosity and effective temperature of the host star. This results in an inner boundary of $0.11 \mathrm{AU}$ and an outer boundary of slightly above $0.21 \mathrm{AU}$. To determine which of GJ 581's planets are located in the HZ, we calculate their equilibrium temperatures $T_{\text {eq }}$ following the methods of Selsis et al. (2007):

$$
T_{\mathrm{eq}}^{4}=\frac{S(1-A)}{f \sigma},
$$

where $S$ is the stellar energy flux, $A$ is the Bond albedo, and $\sigma$ is the Stefan-Boltzmann constant. The redistribution factor $f$ is determined by the efficiency of atmospheric heat redistribution efficiency and is set to 2 for a hot dayside and to 4 for even heat redistribution. Table 2 shows the calculated equilibrium temperatures for the planets in the GJ 581 system assuming an Earth Bond albedo of 0.29 in each case. We look at two different scenarios of the geometry of the GJ 581 system below.

Scenario 1. We take orbital parameters from Mayor et al. (2009). Figure 3 (left panel) depicts this scenario of the geometry of the GJ 581 system with its HZ shown as a gray shaded region. Planet d spends part of its orbit in the HZ. It should be noted that due to the non-zero orbital eccentricity, $T_{\text {eq }}$ is a function of time (or phase angle). Thus, the $T_{\mathrm{eq}}^{f=4}$ for planet d varies from $229 \mathrm{~K}$ at periastron to $154 \mathrm{~K}$ at apastron, causing it to periodically dip into and out of the HZ.

Scenario 2. We take all orbital parameters from Vogt et al. (2010), motivated by the discussion in Anglada-Escudé et al. (2010), which points out that the RV signal of planet d's eccentric orbit could, in fact, be due to two circular orbits of
Table 2

Equilibrium Temperatures for the GJ 581 System Planets

\begin{tabular}{lcrc}
\hline \hline Planet & $a(\mathrm{AU})$ & $T_{\mathrm{eq}}^{f=4}(\mathrm{~K})$ & $T_{\mathrm{eq}}^{f=2}(\mathrm{~K})$ \\
\hline $\mathrm{b}$ & 0.041 & $418 \pm 3$ & $498 \pm 3$ \\
$\mathrm{c}$ & 0.070 & $320 \pm 2$ & $381 \pm 2$ \\
$\mathrm{~d}$ & 0.220 & $181 \pm 1$ & $215 \pm 2$ \\
$\mathrm{e}$ & 0.030 & $489 \pm 2$ & $582 \pm 3$ \\
$\mathrm{f}$ & 0.758 & $97 \pm 1$ & $116 \pm 1$ \\
$\mathrm{~g}$ & 0.146 & $222 \pm 2$ & $264 \pm 2$ \\
\hline
\end{tabular}

Notes. Equilibrium temperatures based on the equations in Selsis et al. (2007) and assuming orbital element values from Mayor et al. (2009, planets b-e) and Vogt et al. (2010, planets $\mathrm{f}$ and g). $f=4$ implies perfect energy redistribution efficiency on the planetary surface, $f=2$ means no energy redistribution. For details, see Section 4 and Figure 3. Note that the quoted uncertainties are based only on the uncertainties in the calculation of GJ 581's luminosity, and not on uncertainties in the orbital elements. As such, they should be regarded as lower limits.

planets $d$ and $g$. The geometry of this scenario is illustrated in Vogt et al. (2010, Figure 6 and Table 2) and shown here in the right panel of Figure 3. In this scenario, planet $\mathrm{g}$ spends all its circular orbit in the HZ with a semimajor axis of $a_{g}=0.15 \mathrm{AU}$, while planet $\mathrm{d}$ is right on the outer edge of the $\mathrm{HZ}$ with $a_{d}=0.22 \mathrm{AU}$. For planet g, an even heat redistribution results in $T_{\mathrm{eq}}=222 \mathrm{~K}$ which is slightly less than the value calculated by Vogt et al. (2010) as anticipated from our smaller value for GJ 581's luminosity. We note that $T_{\mathrm{eq}}=222 \mathrm{~K}$ is below the freezing temperature of water, but does not take into account the greenhouse effect heating due to an atmosphere (for comparison: Earth's $T_{\mathrm{eq}}=255 \mathrm{~K}$ ). See Section 6 of Vogt et al. (2010) and particularly Wordsworth et al. (2010) for more detailed elaborations. Planet d's $T_{\mathrm{eq}}=181 \mathrm{~K}$ for $f=4$ when using its semimajor axis in Equation (2).

\section{SUMMARY AND CONCLUSION}

In this Letter, we present directly determined astrophysical parameters of the late-type, multiplanet host star GJ 581, 
observed as part of our ongoing interferometric survey with the CHARA Array of KM dwarfs with and without planets. We find small but significant differences between literature values and our empirical results for GJ 581, which are explicitly shown in Section 3 and Table 1 . We measure a stellar radius that is larger than predicted by theory-based calibrations (3\%) but a slightly lower than predicted $T_{\mathrm{EFF}}(3.5 \%)$, resulting in our calculated luminosity's being lower than the currently used literature value (by $\sim 7 \%$ ). Thus, we confirm the results in Boyajian et al. (2010) that theoretically calculated radii of $\mathrm{M}$ dwarfs are smaller than the directly measured counterparts.

We recalculate the boundaries of the system's HZ based on our new results. An analysis of the equilibrium temperatures of the six planets orbiting GJ 581 confirms that planet $g$ spends all of its orbit inside the HZ. Depending on the assumed eccentricity of planet d's orbit, it periodically dips into and out of the HZ or spends all of its circular orbit on its outer edge (Section 4). The presence of any moderately dense atmosphere around planets $\mathrm{d}$ and particularly g could provide sufficient greenhouse heating of the planetary surface temperatures (Selsis et al. 2007; Wordsworth et al. 2010) such that any existing water could be in liquid form.

Planetary characterization is playing an increasingly important role in exoplanet research. With the exception of directly imaged planets, the large majority of astrophysical exoplanet parameters are functions of stellar parameters, which in turn are, by necessity, calculated on the basis of stellar models. Particularly in the low-mass regime, however, these stellar models lack observational constraints, leading, not necessarily by fault of the models themselves, to uncertainties and sometimes systematic discrepancies between theoretical and observational results, as we present in this Letter. It is thus hard to overstate the importance of "understanding the parent stars." With recent and ongoing improvements in both sensitivity and spatial resolution of near-infrared and optical interferometric data quality, we are able to ameliorate this situation and provide firm, direct measurements of stellar radii and effective temperatures in the low-mass regime.

We express our gratitude to the (semi-)anonymous referee whose comments on GJ 581's surface temperature and on limb-darkening coefficients clearly improved the quality of this manuscript. T.S.B. acknowledges support provided by NASA through Hubble Fellowship grant HST-HF-51252.01 awarded by the Space Telescope Science Institute, which is operated by the Association of Universities for Research in Astronomy, Inc., for NASA, under contract NAS 5-26555. The CHARA Array is funded by the National Science Foundation through NSF grants AST-0606958 and AST-0908253 and by Georgia State University through the College of Arts and Sciences, as well as the W. M. Keck Foundation. This research made use of the SIMBAD literature database, operated at CDS, Strasbourg, France, and of NASA's Astrophysics Data System. This publication makes use of data products from the Two Micron All Sky Survey, which is a joint project of the University of Massachusetts and the Infrared Processing and Analysis Center/California Institute of Technology, funded by the National Aeronautics and Space Administration and the National Science Foundation. This research made use of the NASA/IPAC/NExScI Star and Exoplanet Database, which is operated by the Jet Propulsion Laboratory, California Institute of Technology, under contract with the National Aeronautics and Space Administration.

\section{REFERENCES}

Anglada-Escudé, G., López-Morales, M., \& Chambers, J. E. 2010, ApJ, 709, 168

Baines, E. K., McAlister, H. A., ten Brummelaar, T. A., Sturmann, J., Sturmann, L., Turner, N. H., \& Ridgway, S. T. 2009, ApJ, 701, 154

Baines, E. K., McAlister, H. A., ten Brummelaar, T. A., Turner, N. H., Sturmann, J., Sturmann, L., Goldfinger, P. J., \& Ridgway, S. T. 2008, ApJ, 680, 728

Baines, E. K., van Belle, G. T., ten Brummelaar, T. A., McAlister, H. A., Swain, M., Turner, N. H., Sturmann, L., \& Sturmann, J. 2007, ApJ, 661, L195

Baines, E. K., et al. 2010, AJ, 140, 167

Bessel, M. S. 1990, A\&AS, 83, 357

Bessell, M. S. 1995, in The Bottom of the Main Sequence-and Beyond, ed. C. G. Tinney (Berlin: Springer), 123

Beust, H., Bonfils, X., Delfosse, X., \& Udry, S. 2008, A\&A, 479, 277

Bonfils, X., et al. 2005, A\&A, 443, L15

Boyajian, T. S., et al. 2010, arXiv:1012.0542

Bryden, G., et al. 2009, ApJ, 705, 1226

Casagrande, L., Flynn, C., \& Bessell, M. 2008, MNRAS, 389, 585

Chabrier, G., \& Baraffe, I. 2000, ARA\&A, 38, 337

Claret, A. 2000, A\&A, 363, 1081

Cutri, R. M., et al. 2003, The 2MASS All Sky Catalog of Point Sources (Pasadena, CA: IPAC)

Delfosse, X., Forveille, T., Perrier, C., \& Mayor, M. 1998, A\&A, 331, 581

Delfosse, X., Forveille, T., Ségransan, D., Beuzit, J., Udry, S., Perrier, C., \& Mayor, M. 2000, A\&A, 364, 217

Farrington, C. D., et al. 2010, AJ, 139, 2308

Hanbury Brown, R., Davis, J., Lake, R. J. W., \& Thompson, R. J. 1974, MNRAS, 167,475

Hauschildt, P. H., Allard, F., Ferguson, J., Baron, E., \& Alexander, D. R. 1999, ApJ, 525, 871

Hawley, S. L., Gizis, J. E., \& Reid, I. N. 1996, AJ, 112, 2799

Henry, T. J., Kirkpatrick, J. D., \& Simons, D. A. 1994, AJ, 108, 1437

Jao, W., Henry, T. J., Subasavage, J. P., Brown, M. A., Ianna, P. A., Bartlett, J. L., Costa, E., \& Méndez, R. A. 2005, AJ, 129, 1954

Johnson, J. A., \& Apps, K. 2009, ApJ, 699, 933

Jones, B. W., \& Sleep, P. N. 2010, MNRAS, 407, 1259

Kasting, J. F., Whitmire, D. P., \& Reynolds, R. T. 1993, Icarus, 101, 108

Kilkenny, D., Koen, C., van Wyk, F., Marang, F., \& Cooper, D. 2007, MNRAS, 380, 1261

Koen, C., Kilkenny, D., van Wyk, F., Cooper, D., \& Marang, F. 2002, MNRAS, 334, 20

Koen, C., Kilkenny, D., van Wyk, F., \& Marang, F. 2010, MNRAS, 403, 1949

Koen, C., Marang, F., Kilkenny, D., \& Jacobs, C. 2007, MNRAS, 380, 1433

Kóspál, Á., Ardila, D. R., Moór, A., \& Ábrahám, P. 2009, ApJ, 700, L73

Markwardt, C. B. 2009, in ASP Conf. Ser. 411, Astronomical Data Analysis Software and Systems XVIII, ed. D. A. Bohlender, D. Durand, \& P. Dowler (San Francisco, CA: ASP), 251

Mayor, M., et al. 2009, A\&A, 507, 487

Nordström, B., et al. 2004, A\&A, 418, 989

Pickles, A. J. 1998, PASP, 110, 863

Rayner, J. T., Cushing, M. C., \& Vacca, W. D. 2009, ApJS, 185, 289

Rojas-Ayala, B., Covey, K. R., Muirhead, P. S., \& Lloyd, J. P. 2010, ApJ, 720, L113

Schlaufman, K. C., \& Laughlin, G. 2010, A\&A, 519, A105

Selsis, F., Kasting, J. F., Levrard, B., Paillet, J., Ribas, I., \& Delfosse, X. 2007, A\&A, 476, 1373

ten Brummelaar, T. A., et al. 2005, ApJ, 628, 453

Udry, S., et al. 2007, A\&A, 469, L43

Underwood, D. R., Jones, B. W., \& Sleep, P. N. 2003, Int. J. Astrobiol., 2, 289 van Altena, W. F., Lee, J. T., \& Hoffleit, D. 1995, VizieR Online Data Catalog, 1174,0

van Belle, G. T., Ciardi, D. R., \& Boden, A. F. 2007, ApJ, 657, 1058

van Belle, G. T., \& von Braun, K. 2009, ApJ, 694, 1085

van Leeuwen, F. 2008, VizieR Online Data Catalog, 1311, 0

Vogt, S. S., Butler, R. P., Rivera, E. J., Haghighipour, N., Henry, G. W., \& Williamson, M. H. 2010, ApJ, 723, 954

von Braun, K., van Belle, G. T., \& Ciardi, D. R. 2010, American Astronomical Society Meeting Abstracts, 216, 425.07

Weis, E. W. 1993, AJ, 105, 1962

Wordsworth, R. D., Forget, F., Selsis, F., Madeleine, J., Millour, E., \& Eymet, V. 2010, A\&A, 522, A22 